\title{
Evolving Transcription Factor Binding Site Models From Protein Binding Microarray Data
}

\author{
Ka-Chun Wong, Chengbin Peng, and Yue Li
}

\begin{abstract}
Protein binding microarray (PBM) is a highthroughput platform that can measure the DNA binding preference of a protein in a comprehensive and unbiased manner. In this paper, we describe the PBM motif model building problem. We apply several evolutionary computation methods and compare their performance with the interior point method, demonstrating their performance advantages. In addition, given the PBM domain knowledge, we propose and describe a novel method called kmerGA which makes domain-specific assumptions to exploit PBM data properties to build more accurate models than the other models built. The effectiveness and robustness of kmerGA is supported by comprehensive performance benchmarking on more than 200 datasets, time complexity analysis, convergence analysis, parameter analysis, and case studies. To demonstrate its utility further, kmerGA is applied to two real world applications: 1) PBM rotation testing and 2) ChIP-Seq peak sequence prediction. The results support the biological relevance of the models learned by kmerGA, and thus its real world applicability.
\end{abstract}

Index Terms-Genetic algorithm, motif discovery, protein binding microarray (PBM), transcription factor (TF) binding site.

\section{INTRODUCTION}

G ENE expression is primarily regulated by the DNA binding of various modulatory transcription factors (TFs) onto cis-regulatory DNA elements near genes in human and other eukaryotes [1]. Different binding combinations of TFs may result in a gene being expressed in different tissues or at different developmental stages. To fully understand a gene's function, it is essential to identify the TFs that regulate the gene and the corresponding TF binding sites (TFBSs) [2], [3].

Several in silico TFBS sequence pattern learning methods (also known as motif discovery methods) have been proposed;

Manuscript received March 23, 2015; revised July 21, 2015; accepted January 14, 2016. This work was supported in part by the City University of Hong Kong under Project $7200444 / C S$, and in part by the Amazon Web Service Research Grant. The proposed method (kmerGA) is available as MATLAB source codes. Interested parties can contact the corresponding author for potential collaborations. This paper was recommended by Associate Editor Y. Jin.

K.-C. Wong is with the Department of Computer Science, City University of Hong Kong, Hong Kong (e-mail: kc.w@cityu.edu.hk).

C. Peng is with the King Abdullah University of Science and Technology, Thuwal 23955-6900, Saudi Arabia.

Y. Li is with Computer Science and Artificial Intelligence Laboratory Massachusetts Institute of Technology, Boston, MA 02139 USA.

This paper has supplementary downloadable multimedia material available at http://ieeexplore.ieee.org provided by the authors. This includes a PDF file, which shows additional figures and tables not included within the paper itself. The total size of the file is $1.09 \mathrm{MB}$.

Color versions of one or more of the figures in this paper are available online at http://ieeexplore.ieee.org.

Digital Object Identifier 10.1109/TCYB.2016.2519380 for instance, Hughes et al. [4] proposed a Gibbs sampling algorithm called AlignACE, to sample and evaluate different possible motif models using a maximum a priori log likelihood score. Bailey and Elkan [5] have proposed multiple EM for Motif elicitation to optimize the expected value of a statistic related to the information content of motif models. In particular, evolutionary computational methods have been demonstrated promising in motif discovery; for instance, Che et al. [6] have proposed a genetic algorithm to exhaustively search for all possible locations of TFBSs. Multiobjective genetic algorithm has also been proposed by Kaya [7], taking into account the TFBS pattern similarity, length, and support. Expectation-maximization (EM) algorithm has been wrapped inside a genetic algorithm framework for discovering spaced TFBSs by Li [8]. Special gap penalties have also been introduced into genetic algorithm by Liu et al. [9]. Two special genetic algorithm operators, SHIFT and ADJUST, have been proposed to improve BioOptimizer with genetic algorithm by Wei and Jensen [10]. A population clustering evolutionary algorithm has been proposed for diverse TFBS learning by Lones and Tyrrell [11]. Chan et al. [12] applied memetic computing with gradient descents to optimize for a consensus TFBS pattern. Detailed comparisons can be found in the survey by Tompa et al. [13].

On the other hand, protein binding microarray (PBM) was developed to measure the binding preference of a protein to a complete set of $k$-mers in vitro [14]. The PBM data resolution is unprecedentedly high, comparing with the other traditional techniques. It has also been shown to be largely consistent with those generated by in vivo genomewide location analysis (ChIP-Chip and ChIP-Seq) [14]. As a result, researchers have applied the PBM technique onto many TFs, and a large amount of PBM data has been being accumulated and deposited to the UniProbe database [15]. In light of this deluge of quantitative affinity data, traditional approaches that rely on cut-offs are no longer adequate. Robust and probabilistic methods were developed to take into account those quantitative affinity data. In light of that, Seed and Wobble has been proposed as a seed-based approach using rank statistics [14]. RankMotif ++ was proposed to maximize the log likelihood of their probabilistic model of binding preferences [16]. MatrixREDUCE was proposed to perform forward variable selections to minimize the sum of squared deviations [17]. MDScan was proposed to combine two search strategies together, namely word enumeration and positionspecific weight matrix updating [18]. PREGO was proposed to 
maximize the Spearman rank correlation between the predicted and the actual binding intensities of ChIP-Chip data [19]. Herd clustering was proposed for multiple TFBS motif elucidation on PBM data [20].

Nonetheless, to the best of the authors' knowledge, there is not any customized evolutionary algorithm which is specifically designed for TFBS pattern learning on PBM data. Therefore, in this paper, we propose a novel method called kmerGA so that the PBM $k$-mer ranking information can be fully captured. To this end, we apply several optimization algorithms to more than 200 PBM datasets for performance comparison, demonstrating that kmerGA can exploit PBM domain knowledge for evolving TFBS models from PBM data.

\section{PROBLEM DESCRIPTION}

For each PBM dataset of the DNA-binding protein of interest, we are given a set of DNA sequences $\left\{\mathrm{seq}_{1}, \mathrm{seq}_{2}, \ldots, \mathrm{seq}_{n}\right\}$ and the corresponding normalized signal intensity values $\left\{I_{1}, I_{2}, \ldots, I_{n}\right\}$ (e.g., array \#1). Following the PBM data analysis convention, we refer to such type of input dataset as an array in this paper. To extract informative motif data, a sliding window of length $k$ is used to scan each DNA sequence $\operatorname{seq}_{i}$ (and its reverse complement) in order to count and record the normalized signal intensity values for each $k$-mer. Once all the DNA sequences are scanned, a list of normalized signal intensity values is obtained for each $k$-mer that is present in those DNA sequences. The median of the list is calculated as the median signal intensity $\mu_{m}$ for each $k$-mer $s_{m}$. Among those $k$-mers, some are motif instances (positive $k$-mers) while the others are just background $k$-mers. Robust estimate procedures proposed in RankMotif ++ [16] can then be applied to learn the positive $k$-mers. Nonetheless, it has been pointed out that such a robust procedure may not be suitable for all proteins [21]. In light of that, the highly ranked $k$-mers are regarded as the positive $k$-mers in this paper.

After a set of positive $k$-mers were selected, they are aligned using a multiple sequence alignment method such as [22]. The aligned $k$-mers are then input for training a motif matrix model to represent the binding preferences of the DNA-binding protein of interest, using optimization algorithms.

In this paper, we aim at evolving motif models (using evolutionary computation) which can truly capture the information from PBM data, reflecting the true binding sequence preferences of DNA-binding proteins. In particular, we seek to evolve matrix models to accurately rank the median signal intensities of positive $k$-mers. Therefore, the problem is formulated as follows.

Input: A set of aligned positive DNA $k$-mers with their median signal intensities $D=\left\{\left(s_{1}, \mu_{1}\right),\left(s_{2}, \mu_{2}\right)\right.$, $\left.\left(s_{3}, \mu_{3}\right), \ldots,\left(s_{M}, \mu_{M}\right)\right\}$ of length $L$, where $s_{m}$ is the $m$ th aligned DNA $k$-mer with its median signal intensity $\mu_{m}$. Each aligned DNA $k$-mer $s_{m}$ can be represented as $s_{m}=$ $s_{m 1} s_{m 2} \ldots s_{m L}$, where $s_{m p}$ is the $p$ th nucleotide

$$
\begin{aligned}
& s_{m p} \in\{A, C, G, T,-\} \\
& \forall m \in\{1,2, \ldots, M\}, \forall p \in\{1,2, \ldots, L\} .
\end{aligned}
$$

Output: A matrix model $\Theta$ trained to represent the input aligned $k$-mers $D$ such that the Spearman rank correlation coefficient between the predicted scores $\left\{S_{\Theta}\left(s_{m}\right), \forall m \in\right.$ $\{1,2, \ldots, M\}\}$ and the actual median binding intensities $\left\{\mu_{m}, \forall m \in\{1,2, \ldots, M\}\right\}$ of the input aligned $k$-mers $D$ is maximized (i.e., objective function)

$$
\arg \max _{\Theta} f(\Theta, D)=\frac{\sum_{m=1}^{M}\left(\left(X_{m}-\bar{X}\right)\left(Y_{m}-\bar{Y}\right)\right)}{\sqrt{\sum_{m=1}^{M}\left(X_{m}-\bar{X}\right)^{2} \sum_{m=1}^{M}\left(Y_{m}-\bar{Y}\right)^{2}}}
$$

under the following sparse linear constraints:

$$
\begin{aligned}
\sum_{i \in\{A, C, G, T,-\}} \Theta_{i j} & =1 \quad \forall j \in\{1,2, \ldots, L\} \\
0 \leq \Theta_{i j} & \leq 1 \quad \forall i \in\{A, C, G, T,-\}, \forall j \in\{1,2, \ldots, L\}
\end{aligned}
$$

where $X_{m}$ is the rank of $S_{\Theta}\left(s_{m}\right)$ and $Y_{m}$ is the rank of $\mu_{m}$. $\bar{X}$ and $\bar{Y}$ are the average ranks and the function $S_{\Theta}\left(s_{m}\right)$ is defined as follows:

$$
S_{\Theta}\left(s_{m}\right)=\log \frac{\prod_{j=1}^{L} \prod_{i=\{A, C, G, T,-\}} \Theta_{i j}^{\left[s_{m j}=i\right]}}{\prod_{j=1}^{L} \prod_{i=\{A, C, G, T,-\}} B_{i}^{\left[s_{m j}=i\right]}}
$$

where $B_{i}$ is the occurring fraction of the $i$ th nucleotide in all the background sequences [23].

\section{Methodology}

In this paper, we have introduced a motif model building problem which is to rank the top $k$-mers from PBM. We note that past literature usually focus on recognizing motif consensus patterns which is not our major focus here. We focus on evolving good models to fit regressions on the ranks of the top $k$-mers from PBM. To obtain the models needed, we apply the existing methods as well as propose and apply a new evolutionary algorithm (kmerGA) to PBM benchmark datasets.

\section{A. Representation}

To represent each candidate TFBS sequence pattern, we adopt the position frequency matrix (PFM) representation which is the de facto TFBS model standard [23]. Each TFBS sequence pattern of width $w$ is represented as a 4-by- $w$ matrix $\Theta$. The $j$ th column corresponds to the $j$ th position of the underlying TFBS, whereas the $i$ th row of $\Theta$ corresponds to the $i$ th biological character. In the context of DNA sequences, we have four characters $\{A, C, G, T\}$. Therefore, $\Theta_{i j}$ is the occurring fraction of the $i$ biological character at the $j$ th position; for example, the PFM of the SOX9 protein is tabulated as follows:

$\Theta_{\operatorname{sox} 9}=\begin{gathered}A \\ C \\ C \\ G \\ T\end{gathered}\left(\begin{array}{ccccccccc}0.31 & 0.68 & 0.75 & 0.01 & 0.83 & 0.89 & 0.06 & 0.31 & 0.13 \\ 0.10 & 0.09 & 0.06 & 0.91 & 0.06 & 0.04 & 0.01 & 0.09 & 0.13 \\ 0.40 & 0.10 & 0.01 & 0.04 & 0.01 & 0.03 & 0.03 & 0.49 & 0.69 \\ 0.19 & 0.13 & 0.18 & 0.04 & 0.10 & 0.04 & 0.90 & 0.11 & 0.05\end{array}\right)$.

We can observe that the nearly invariant positions of the SOX9 motif are the fourth and seventh position. At the fourth position, cytosine (C) is found to be dominant while guanines $(\mathrm{G})$ and thymines $(\mathrm{T})$ have just been found few times. 


\section{B. Scoring Scheme}

As mentioned in the problem description, our objective is to maximize the Spearman rank correlation coefficient $f(\Theta, D)$ between the predicted binding affinity and the actual binding affinity. Therefore, the scoring scheme in this paper is the Spearman rank correlation coefficient $f(\Theta, D)$. After each run of each method, within the final population $\left\{\Theta_{1}, \Theta_{2}, \ldots, \Theta_{N}\right\}$, we select the individual $\Theta_{k}$ such that $f\left(\Theta_{k}, D\right) \geq f\left(\Theta_{i}, D\right) \forall i \in$ $\{1,2, \ldots, N\}$. The value of $f\left(\Theta_{k}, D\right)$ is reported to be the final Spearman rank correlation coefficient achieved by that run.

In the following sections, we will have multiple runs for each method on each dataset. Therefore, the mean and standard deviation of the final Spearman rank correlation coefficients will be computed to summarize the multiple runs' performance of each method on each dataset. To statistically quantify the significance of the performance difference, $t$-tests are computed to observe the performance difference between the proposed method (kmerGA) and the other methods on each dataset.

\section{Existing Methods' Description}

Several existing methods have been applied to the proposed problem (see Section II), including interior point method (IPM) [24], genetic algorithm (GA) [25], differential evolution (DE) [26], crowding genetic algorithm (CGA) [27], and crowding differential evolution (CDE) [27]. Those methods are selected to represent different algorithmic paradigms; for instance, interior point method represents the line search optimization paradigm [28]; genetic algorithm represents the nature-inspired optimization paradigm [29]; differential evolution represents the stochastic beam search optimization paradigm [30], [31]; and crowding differential evolution and crowding genetic algorithm represent the multimodal optimization paradigm [32].

\section{Proposed Approach (kmerGA)}

The previously described methods are generic ones which can be widely applied to different problems. Nonetheless, they have not taken advantages of specific domain knowledge. In the context of PBM data, we would like to note that the sequence context and numerical locality have not been fully explored by those generic methods. To take into account those PBM-data-specific features, we propose a novel genetic algorithm (kmerGA) which reproductive operators can fully explore such features as shown in Fig. 1.

1) Block Crossover: It has been reported that TFBSs usually consist of binding cores, controlling its sequence specificity [33]. To promote effective binding core exchanges during evolution, it is intuitive to propose a block crossover operator which can exchange the binding core information between individuals as shown in Fig. 1. A random continuous block is exchanged between individuals to give birth to offspring. To handle boundary, circular blocks can be exchanged. There are two control parameters for block crossovers: 1) the block starting column index and 2) block width. In this paper, we randomly choose the starting column index and width uniformly.

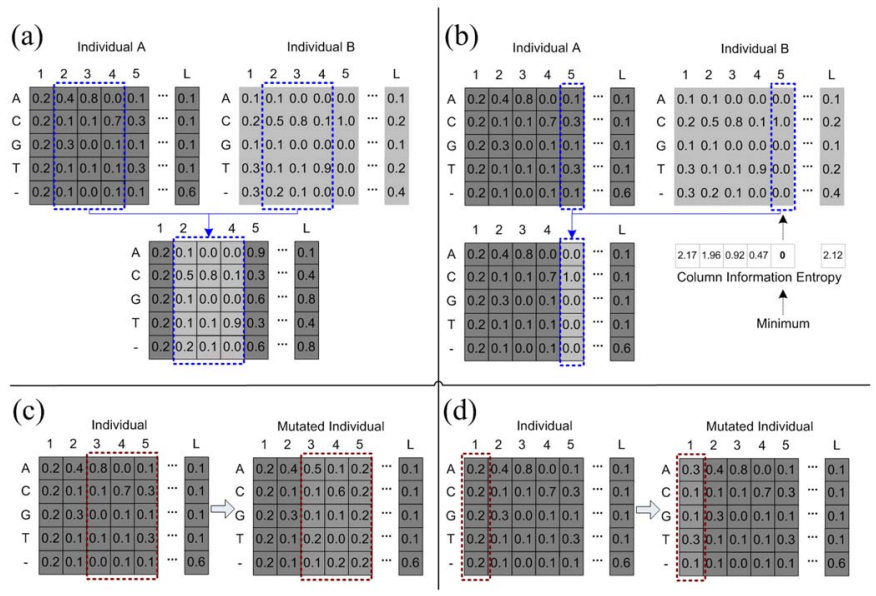

Fig. 1. Proposed crossover and mutation operators of kmerGA. (a) Block crossover. (b) Sequence context crossover. (c) Block mutation. (d) Column mutation.

2) Sequence Context Crossover: In addition to block crossovers, we would like to note that some positions of the existing TFBSs are highly conserved (i.e., low entropy) [34]. To exploit such a feature, a sequence context crossover operator is proposed as shown in Fig. 1. In that operator, two individuals are chosen based on the parent selection scheme given. One individual becomes the base (e.g., individual A in Fig. 1) while the columns of the other individual are examined for their information entropy (e.g., individual B in Fig. 1). The column with the lowest information entropy is chosen for column swap between the two individuals. The rationale behind it is that we assume conserved (low entropy) positions are rare in nature. If it can be found, it means that it has undergone sufficient positive selection over a long period of evolution, reflecting its functionally important role in TFBSs [34].

3) Intermediate Crossover: The previous two crossover operators are all based on block exchanges. They are very suitable for diversity maintenance and global exploration, but not for immediate convergence. To remedy that, the classic intermediate crossover operator is adopted in the proposed approach [35]. Briefly, an offspring can be generated by taking the average values of two individuals.

4) Block Mutation: Similar to block crossover, block mutation is inspired from the existing knowledge on TFBS binding cores [33]. In a block mutation, a random block is chosen as shown in Fig. 1. All of the elements inside the chosen block are mutated simultaneously. The idea is actually similar to the hypermutation operators proposed in [36]. The major difference is that we limit our mutation range to blocks.

5) Column Mutation: Block mutations are somewhat de novo. In practice, it is hard for a block mutation to succeed in few runs. Thus column mutation is proposed to be practical for incremental convergence as shown in Fig. 1. A random column is chosen and mutated. It can be regarded as a special case of block mutation where block width is set to 1 .

6) Random Mutation: To ensure the explorative power of the proposed method, random mutation is also adopted [35]. In random mutation, each element of matrix model has a 
probability to be mutated in each generation, regardless of its position in matrix model.

\section{E. Time Complexity Analysis}

In this section, we focus on the time complexity of the proposed approach (kmerGA) since the complexities of the other methods used have been well-studied in the past [35].

For parent selection, stochastic uniform selection is used, resulting in linear complexity $O(N)$ where $N$ is the population size used. Once parents are selected, the crossover operators are applied to each parent pair. In essence, the bottleneck of the crossover operators in this paper is the sequence context crossover operation while the others are just block exchanges (i.e., block crossover) and simple algebraic operation (i.e., intermediate crossover) which linearly scales with the sizes of the models $(O(5 L))$. On the other hand, sequence context crossover involves an additional step to calculate the column information entropies, which results in another time complexity $O(5 L)$. In total, each sequence context crossover costs $O(5 L+5 L)$ which is still linear. After the crossover operations, mutation operators are applied. From Fig. 1, it is trivial that they also incur linear complexity to the model sizes $O(5 L)$. Thus the parent selection and offspring generation have a linear complexity $O(L N)$ in total.

In the fitness function evaluation procedure, for each individual, we have to compute $S_{\Theta}\left(s_{m}\right)$ for all the $M$ positive $k$-mers, resulting in $O(5 M L)$. Merge-sort is applied to compute the rank $X_{m}$ of each $S_{\Theta}\left(s_{m}\right)$ which costs $O(M \log M)$. After the ranks are computed, only linear summation operations are applied, costing $O(M)$ for all the $M$ positive $k$-mers. Therefore, each function evaluation costs $O(M L+M \log M+M)$ which can be simplified as $O(M L+M \log M)$. Given that we have $N$ offspring individuals, $O(N M L+N M \log M)$ is thus counted as the overall time complexity of the fitness function evaluation procedure.

After the fitnesses have been computed, truncation is used as survival selection [35]. Merge-sort is applied to truncate the whole population in each iteration. Thus a time complexity of $O(N \log N)$ is expected.

In summary, each iteration of kmerGA costs $O(L N+N M L+$ $N M \log M+N \log N)$ in time complexity.

\section{EXPERIMENTS}

\section{A. Data Sources}

We have adopted the PBM datasets from [16]. Specifically, the PBM datasets focus on five proteins of interest. For each protein, we have two array sets of DNA probe sequences, i.e., arrays \#1 and \#2. Each DNA probe sequence on the array is associated with a normalized signal intensity value. The higher the value, the higher is the binding preference of a DNA-binding protein to that DNA sequence. For each DNAbinding protein, the two arrays (data replicates) are both used for performance comparison. In particular, we seek to examine how each method can rank the aligned input $k$-mers accurately using the fitness function (i.e., the Spearman rank correlation coefficient in Section II).

\section{B. Parameter Setting}

All methods are implemented in MATLAB codes with the default floating point number representation. Progressive multiple alignment is adopted; pair-wise distances between sequences are computed by counting the proportion of sites at which each pair of sequences are similar and different using NUC44 (ignoring gaps) [37]. Assuming equal variance and independence of evolutionary distance estimates, the guide tree is calculated by the neighbor-joining method. For all evolutionary computation methods, population type is overlapping [35]. Population size is set to 50. On each dataset, we have run each method 30 times to collect enough performance measurements for statistical tests (i.e., $t$-tests). Regarding about the termination condition, as mentioned in the previous study [38], different algorithms perform different operations in one generation, it is unfair to set the termination condition as the number of generations. Alternatively, it is also unfair to adopt CPU time because it substantially depends on the implementation techniques for different algorithms; for instance, the sorting techniques to find elitists and the programming languages used. In contrast, fitness function evaluation is always the performance bottleneck [39]. ${ }^{1}$ Thus the termination condition is set to 1000 fitness function evaluations in this paper. The uniform nucleotide background distribution from PBM is adopted. Numerical finite difference method is adopted to approximate gradients for the interior point method. For all genetic algorithm methods except kmerGA, block crossover and random mutation are adopted. For kmerGA, all of the previously described crossover and mutation operators are used. For each crossover, we draw a random value $[0,1]$ to see which crossover and mutation operators are used $(0.2$ for block crossover, 0.2 for sequence context crossover, and 0.6 for intermediate crossover; 0.2 for block mutation, 0.2 for column mutation, and 0.6 for random mutation.). For all genetic algorithm methods, crossover probability is set to 0.8 while mutation probability is set to 0.05 . Gaussian mutation with step size 0.5 is applied [35]. Parent selection is set to stochastic uniform selection. For Genetic Algorithm (GA), roulette-wheel (also known as fitness proportional) selection is used for survival selection; for Crowding Genetic Algorithm (CGA), crowding selection is used for survival selection; for kmerGA, truncation is used for survival selection. For all differential evolution methods, crossover probability is set to 0.8 while the trial vector coefficient $F$ is set to 0.9 . For crowding-based methods, crowding factor is set to the population size, avoiding replacement errors.

\section{Performance Comparison}

We have run each method on each dataset for 30 times. For each run, the fittest individual model is selected as the final model. For each dataset, we have calculated the mean and standard deviation of the 30 runs for each method as shown in Table I. From the table, it is observed that kmerGA performs the best among the six methods. In particular, kmerGA consistently achieves the highest means, reflecting that kmerGA is

\footnotetext{
${ }^{1}$ For instance, over $10 \mathrm{~h}$ are needed to evaluate a calculation in computational fluid dynamics [40].
} 
TABLE I

Performance Comparison: Spearman Rank Correlation Coefficients Between the Actual Median Binding Intensities of the Input Aligned $k$-mers and the Tentative Scores Predicted by Different Methods (on the Top Row) On DifFerent PBM Datasets (on the Leftmost Column). The Highest Means Are Highlighted in Bold. For Each Dataset, a $t$-Test Is Conducted to Observe the Statistical Significance of the Pair-Wise Performance Differences Between KmerGa and the Best of the Other Methods Because We Observe Performance Normality. If $p$-Value Is Less Than 0.05, a Tick $(\checkmark)$ Is Shown

\begin{tabular}{l|ccccccc}
\hline \hline dataset \method & IPM & GA & CGA & DE & CDE & kmerGA & $p<0.05$ \\
\hline \hline Cbf1_deBruijn_v1 & $0.07 \pm 0.18$ & $0.31 \pm 0.08$ & $0.69 \pm 0.05$ & $0.74 \pm 0.04$ & $0.69 \pm 0.03$ & $\mathbf{0 . 8 0} \pm \mathbf{0 . 0 2}$ & $\checkmark$ \\
Cbf1_deBruijn_v2 & $0.12 \pm 0.17$ & $0.44 \pm 0.05$ & $0.68 \pm 0.03$ & $0.70 \pm 0.02$ & $0.67 \pm 0.03$ & $\mathbf{0 . 7 5} \pm \mathbf{0 . 0 2}$ & $\checkmark$ \\
Ceh-22_deBruijn_v1 & $0.15 \pm 0.17$ & $0.32 \pm 0.03$ & $0.51 \pm 0.02$ & $0.52 \pm 0.02$ & $0.50 \pm 0.02$ & $\mathbf{0 . 5 3} \pm \mathbf{0 . 0 3}$ & \\
Ceh-22_deBruijn_v2 & $0.09 \pm 0.24$ & $0.35 \pm 0.04$ & $0.58 \pm 0.02$ & $0.59 \pm 0.02$ & $0.56 \pm 0.03$ & $\mathbf{0 . 6 1} \pm \mathbf{0 . 0 3}$ & $\checkmark$ \\
Oct-1_deBruijn_v1 & $0.08 \pm 0.11$ & $0.14 \pm 0.08$ & $0.43 \pm 0.04$ & $0.44 \pm 0.04$ & $0.37 \pm 0.03$ & $\mathbf{0 . 5 5} \pm \mathbf{0 . 0 4}$ & $\checkmark$ \\
Oct-1_deBruijn_v2 & $0.14 \pm 0.15$ & $0.26 \pm 0.08$ & $0.49 \pm 0.02$ & $0.51 \pm 0.02$ & $0.48 \pm 0.02$ & $\mathbf{0 . 5 4} \pm \mathbf{0 . 0 2}$ & $\checkmark$ \\
Rap1_deBruijn_v1 & $0.12 \pm 0.17$ & $0.38 \pm 0.04$ & $0.63 \pm 0.03$ & $0.65 \pm 0.03$ & $0.64 \pm 0.03$ & $\mathbf{0 . 6 8} \pm \mathbf{0 . 0 4}$ & $\checkmark$ \\
Rap1_deBruijn_v2 & $0.10 \pm 0.15$ & $0.36 \pm 0.06$ & $0.58 \pm 0.04$ & $0.62 \pm 0.03$ & $0.59 \pm 0.04$ & $\mathbf{0 . 6 6} \pm \mathbf{0 . 0 5}$ & $\checkmark$ \\
Zif268_deBruijn_v1 & $0.07 \pm 0.09$ & $0.16 \pm 0.07$ & $0.48 \pm 0.06$ & $0.49 \pm 0.05$ & $0.43 \pm 0.05$ & $\mathbf{0 . 7 2} \pm \mathbf{0 . 0 3}$ & $\checkmark$ \\
Zif268_deBruijn_v2 & $0.08 \pm 0.11$ & $0.20 \pm 0.08$ & $0.57 \pm 0.05$ & $0.58 \pm 0.04$ & $0.51 \pm 0.05$ & $\mathbf{0 . 6 6} \pm \mathbf{0 . 0 4}$ & $\checkmark$ \\
\hline
\end{tabular}

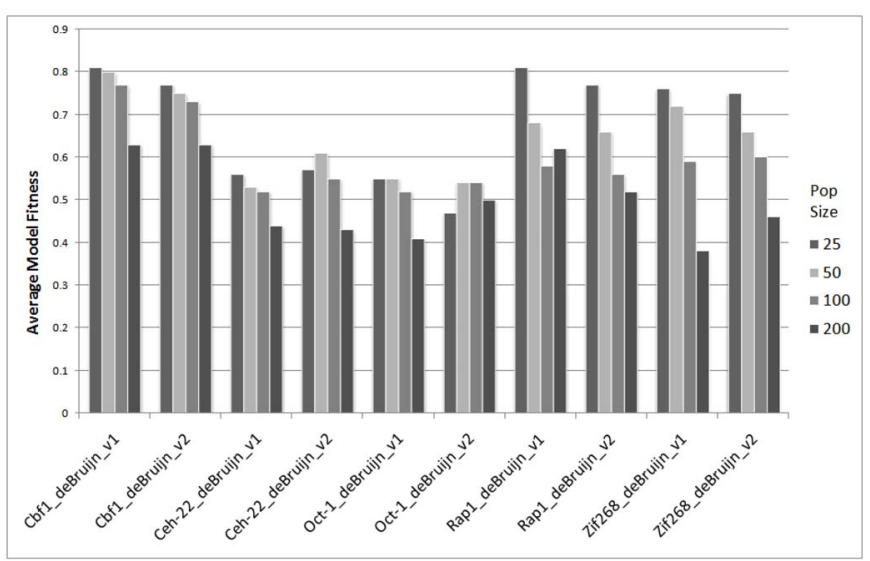

Fig. 2. Parameter analysis on population size of kmerGA under 1000 fitness function evaluations. The horizontal axis denotes different PBM datasets, while the vertical axis denotes the mean performance of kmerGA to recover the ranks of the input aligned $k$-mers in terms of Spearman rank correlation coefficient. Different gray colors denote different population sizes.

competitive at recovering the ranks of the $k$-mers on different PBM datasets. To assess its performance rigorously, we have computed $t$-tests to ascertain that the performance of kmerGA is different from the others with sound statistical significance $(p<0.05)$. It is observed that kmerGA performance is statistically different from the other methods on all datasets except Ceh-22_deBruijn_v1. Another observation is that all evolutionary computing techniques show better performance than the state-of-the-art numerical technique (IPM) which has additional fitness evaluations on gradient approximation.

\section{Parameter Analysis}

To access the robustness of kmerGA, a parameter analysis is conducted. Similar to other evolutionary methods, population size is an important parameter for kmerGA. Thus we have rerun kmerGA on the previous datasets to see how the performance of kmerGA is changed with population size. In particular, population is varied from 25 to 200 under the same number of fitness evaluations (NFEs). The results are depicted in Fig. 2. It can be observed that kmerGA usually works best with small population sizes on most datasets under the same NFEs, even for population size $=25$. Its performance is degraded as the population size is increased. The results are

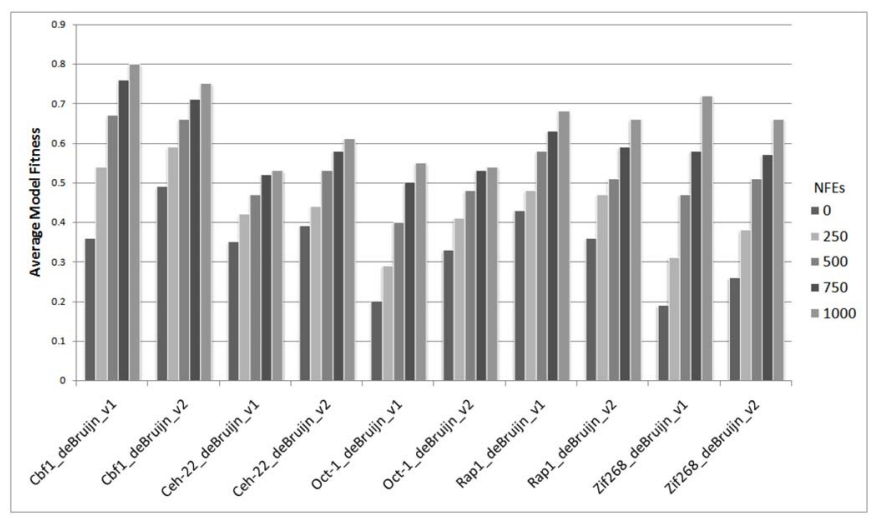

Fig. 3. Convergence behavior of kmerGA. The horizontal axis denotes different PBM datasets, while the vertical axis denotes the mean performance of kmerGA to recover the ranks of the input aligned $k$-mers in terms of Spearman rank correlation coefficient. Different gray colors denote different NFEs.

interesting because it implies that the performance of kmerGA can be improved further from the results tabulated in Table I. On the Ceh-22 and Oct-1 datasets, kmerGA can still work very well with population size $=50$.

\section{E. Convergence Behavior}

To observe the convergence behavior of kmerGA, a convergence analysis is conducted. The NFEs is varied to observe the convergence behavior of kmerGA as shown in Fig. 3. It can be observed that a monotonically fitness-increasing trend is shared across the PBM datasets, although their convergence rates vary on a case by case basis.

\section{F. Extended Performance Comparison}

To demonstrate the performance of kmerGA further, all the methods were run and tested on the comprehensive mouse PBM dataset repository [41], which consists of 230 PBM array datasets. The results are tabulated in Tables A1-A4 of supplementary material. It can be observed that, although the benchmark datasets have been comprehensively expanded, kmerGA still performs the best on nearly all of the datasets with statistical significances, demonstrating its superior performance. In addition, except kmerGA, we observe that the canonical DE algorithm is the best method among the other methods in this paper. It may imply that stochastic beam search methods using 


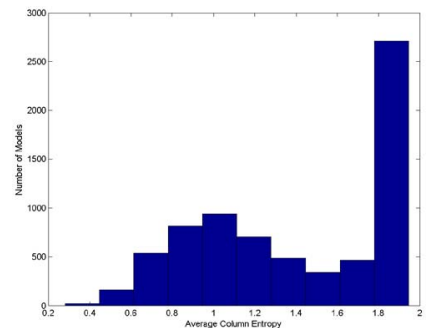

(a)

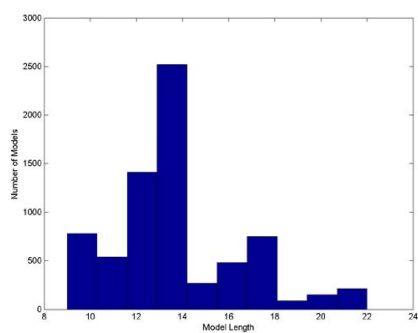

(b)
Fig. 4. Model distributions sorted by (a) average column entropy and (b) model length.

arithmetic operators (e.g., DE) can work well for the problem proposed in this paper, in contrast to block exchange methods (e.g., GA) and single line search methods (e.g., IPM).

If we relax our termination condition to be tenfold (i.e., 10000 fitness function evaluations), their performance may be different. Thus we have conducted additional experiments on the same datasets with the relaxed termination condition. The results are tabulated in Tables A5-A8 of the supplementary material. It can be observed that kmerGA can still achieve the best performance on more than 200 datasets, demonstrating its unique effectiveness. Another interesting observation is that CGA stands out among the other methods while the canonical DE algorithm performs relatively less competitive than before. A possible explanation is that the relaxed termination condition fully unleash the multimodal optimization ability of CGA, enhancing its global optimization ability in this paper. It is not surprising since such a phenomenon is also observed in the previous numerical multimodal optimization studies [38].

\section{G. Model Analysis}

After the runs (30 runs for 240 PBM datasets), we have learned thousands of models. It is interesting for us to investigate how the models have been distributed. In particular, besides model fitnesses in Tables A1-A8, we are also interested in the average column entropies and lengths of the models as shown in Fig. 4. It can be observed that the average column entropies of the models tend to be polarized to the highest values, reflecting that the sequence patterns we have learned are very complex from the information theory view. On the other hand, it can be observed that the model lengths are usually around 12-14 nt which are consistent with the existing TFBS knowledge [21].

\section{H. Case Studies}

1) Tri-Helical Helix-Turn-Helix Domain: The tri-helical helix-turn-helix (tHTH) domain family is a DNA-binding domain composed of three alpha helices joined by short strand of amino acids. It has been found in hundreds of DNAbinding proteins from both eucaryotes and procaryotes. tHTH domains usually act in dimers. As an illustrative example, the Mybl1 protein is selected and studied in depth. In particular, we are interested in the models we have learned from its PBM datasets using kmerGA. Thus we have aggregated the 30 models we have learned from its 30 runs and depicted its sequence logo as shown in Fig. 5. It can be observed that the sequence logo consists of two reverse complement motifs (AAC and GTT), reflecting that the Mybl1 protein uses the dimeric binding mechanism to bind to its binding sites. It is consistent with the previous observation that some tHTH proteins bind to DNA in dimeric forms. To investigate it further, we have plotted a boxplot and cumulative distribution function (CDF) plots for the column entropies at different positions of the models we have learned on the Myb1 dataset in the same figure (i.e., Fig. 5). Interestingly, it can be observed that the dimeric binding phenomenon can still be observed; for instance, the column entropy of the dimeric hinge column position (i.e., position 9) is found lower than those of the other binding column positions (i.e., positions 6-8 for the AAC motif and positions 10-12 for the GTT motif). Another interesting observation is that the nucleotide fractions of the models have correlations with their model fitnesses as shown in Fig. 5. It can be observed that the adenine fractions of the models are positively correlated to the models' fitnesses (Spearman rank correlation coefficient $=0.63$ with $p=0.00019)$. In contrast, the guanine fractions of the models are negatively correlated to the models' fitnesses (Spearman rank correlation coefficient $=-0.51$ with $p=0.0043$ ). A possible explanation is that adenines are usually found near core binding column positions which encourage their occurrences for high model fitnesses, while the role of guanine is the opposite.

2) Winged Helix Turn Helix Domain: The winged helix turn helix (wHTH) domain family is a DNA-binding domain which has three alpha helices and four beta strands (e.g., PF03444). Especially, the domain is characterized by the alternative intervention between the helices and strands. A schematic of wHTH is shown in PDB with the code "4IRI" in which a crystal structure of the auto-inhibited ERG ETS domain-DNA complex with the wHTH domain can be found. Our previous computational experiments include several proteins with tHTH domains. As an illustrative example, the Foxa2 protein is selected and studied in depth. In particular, we are interested in the models we have learned from its PBM datasets using kmerGA. Thus we have aggregated the 30 models we have learned from its 30 runs and depicted its sequence logo as shown in Fig. 6. In contrast to the Myb1 protein, Foxa2 binds to DNA in monomer fashion. Thus we can observe that the sequence logo of Foxa2 is not composed of two reverse complement motifs. Instead, it is just a single motif which core binding length spans across $8 \mathrm{nt}$. To investigate it further, we have plotted a boxplot and CDF plots for the column entropies at different positions of the models we have learned on the Foxa2 dataset in the same figure (i.e., Fig. 6). Interestingly, it can be observed that the column entropy distribution is different from the Mybl1. Different columns have different entropy distribution, reflecting the monomeric binding nature of Foxa2. In addition, we observe that positions 5 and 8 are more conserved across the model population than the others. It gives insights into how the Foxa2 protein binds to DNA. For the sake of completeness, we have also plotted the scatter plots for the relationship between nucleotide fractions and model fitnesses on the Foxa 2 dataset. None of the nucleotides are found statistically correlated to model fitnesses. 


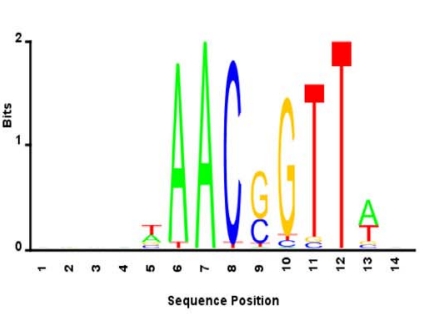

(a)

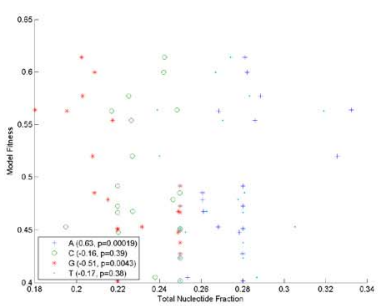

(b)

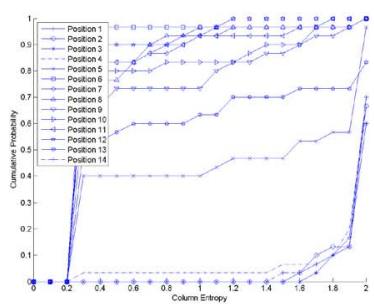

(c)

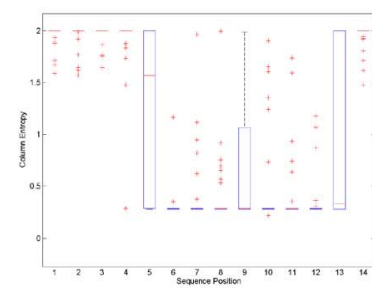

(d)

Fig. 5. Visualization of the models learned on the Mybl1 dataset using kmerGA. (a) Sequence logo. (b) Model fitness versus nucleotide fraction. (c) CDF of column entropy at different column positions. (d) Boxplot on column entropy at different column positions.

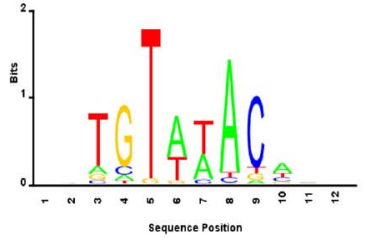

(a)

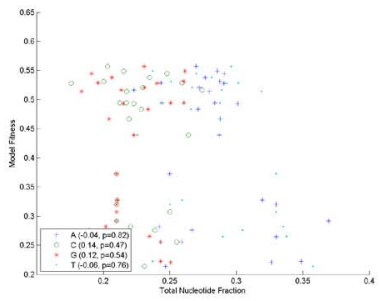

(b)

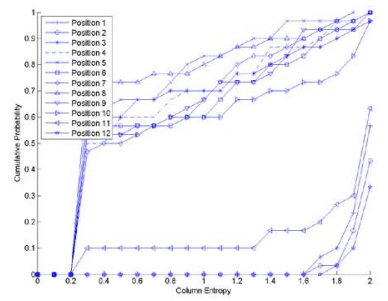

(c)

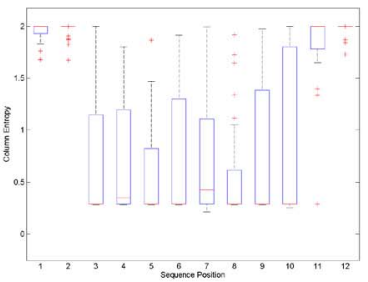

(d)

Fig. 6. Visualization of the models learned on the Foxa2 dataset using kmerGA. (a) Sequence logo. (b) Model fitness versus nucleotide fraction. (c) CDF of column entropy at different column positions. (d) Boxplot on column entropy at different column positions.

3) Basic Helix-Loop-Helix Domain: The basic helix-loophelix (bHLH) domain family is a DNA-binding domain which consists of two alpha helices connected by a loop structure (e.g., PF00010). In most cases, they bind to DNA as a dimer (i.e., two domains). A schematic of bHLH can be found using the PDB code "2QL2." Similar to wHTH, the proteins with bHLH domains should bind to two motifs. Thus we have searched through the PBM datasets and investigated the bHLH matrix models we have learned. As an illustrative example, the Bhlhb2 protein is selected. Similar to the previous domains, its models learned are depicted in Fig. A2 of the supplementary material. We can observe the phenomenon similar to the Mybl1 protein. The matrix models we have learned are composed of two (reverse) complement DNA motifs (CAC and GTG), indicating that the Bhlhb2 binds to DNA in a dimeric fashion. It is supported by the entropy analysis plots further in the same figure. Similar to the previous proteins, the neighboring positions (i.e., positions 1 and 10) are high in entropy, which implies their lesser roles in binding. Regarding about the nucleotide fractions, we can observe that there is a positive correlation between cytosine fractions and model fitnesses with statistical significances $(p=0.0074)$.

4) SAND Domain: The SAND (Sp100, AIRE-1, NucP41/75, DEAF-1) domain family is commonly found in nuclear proteins. It functions in chromatin-dependent transcription control with its DNA-binding modular structure which consists of a strongly twisted, five-stranded antiparallel beta-sheet with four alpha-helices packing against one side of the beta-sheet (e.g., IPR000770). A schematic of SAND domain can be found using the PDB code "1OQJ." Unlike the previous domains, SAND domain is a relatively unknown domain. Thus we have searched through the PBM datasets and investigated the SAND matrix models we have learned.

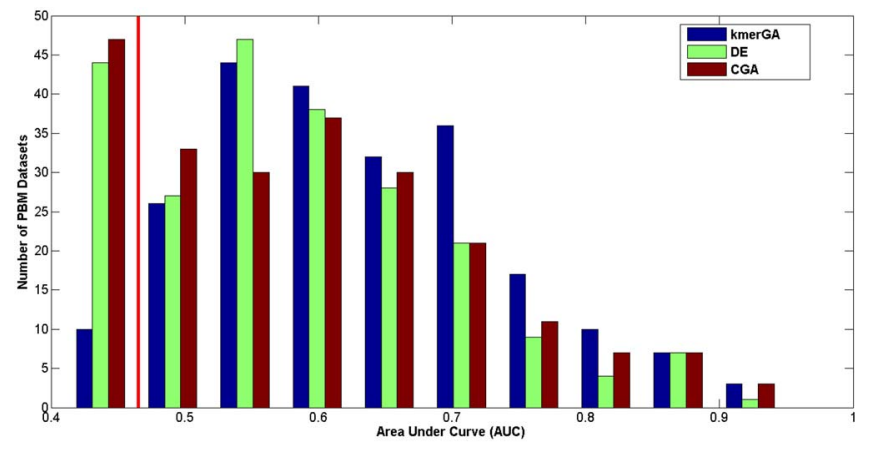

Fig. 7. AUC value distribution after PBM rotation testing. The vertical line is the baseline borderline ( $\mathrm{AUC} \geq 0.5$ ).

As an illustrative example, the Gmeb1 protein is selected. Similar to the previous domains, its models learned are depicted in Fig. A3 of the supplementary material. We can observe the phenomenon similar to the Foxa2 protein, indicating that the Gmeb1 may function as a monomer for DNA binding. In addition, we observe that positions 5 and 8 are more conserved across the model population than the others. It gives insights into which nucleotides the Gmeb1 protein binds to for specificity-determining binding. From the scatter plots for the relationships between nucleotide fractions and model fitnesses on the Gmeb1 dataset, we can observe that the adenine fractions of the models are positively correlated to the models' fitnesses (Spearman rank correlation coefficient $=0.39$ with $p=0.032$ ). In contrast, the guanine fractions of the models are negatively correlated to the models' fitnesses (Spearman rank correlation coefficient $=-0.44$ with $p=0.015$ ). A possible explanation is that guanines are usually found in the noncore binding column positions (i.e., positions 5 and 8), discouraging their occurrences for high model fitnesses, while the role of adenine is the opposite. 


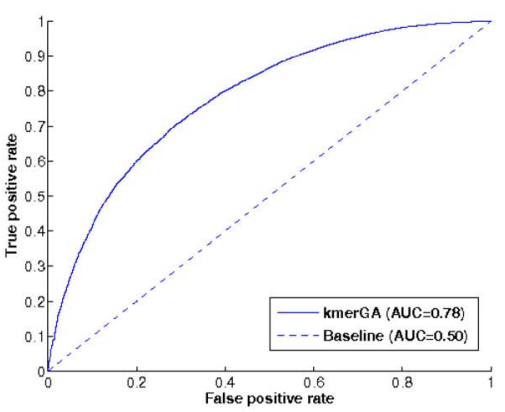

(a)

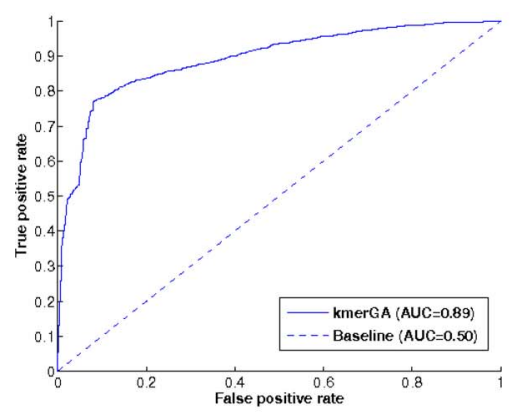

(b)

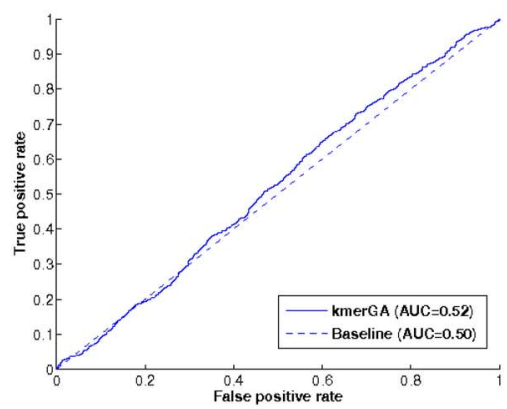

(c)

Fig. 8. Peak sequence prediction performance kmerGA on the ENCODE peak sequence datasets (K562 cell line). Different thresholds are cut at the prediction scores $B_{M}(D)$ to observe the performance tradeoff between sensitivity (true positive rate) and false positive rate of kmerGA on (a) Egr1 ChIP-Seq dataset, (b) Max ChIP-Seq dataset, and (c) Zbtb3 ChIP-Seq dataset.

5) $\mathrm{C} 2 \mathrm{H} 2$ Zinc Finger Domain: The $\mathrm{C} 2 \mathrm{H} 2$ zinc finger $(\mathrm{C} 2 \mathrm{H} 2 \mathrm{ZF})$ domain family is the most characterized type of zinc finger domains. It is commonly found in mammalian TFs. It has diverse functions in regulating gene expressions. It adopts a simple beta-beta-alpha fold with a specific protein sequence motif with two cysteines and two histidines. A schematic of $\mathrm{C} 2 \mathrm{H} 2 \mathrm{ZF}$ domain can be found using the PDB code "2JPA." The distinguishing feature of $\mathrm{C} 2 \mathrm{H} 2 \mathrm{ZF}$ domains is that they usually occur in tandem. To estimate the PBM data capability, we have searched through the PBM datasets and investigated the $\mathrm{C} 2 \mathrm{H} 2 \mathrm{ZF}$ matrix models we have learned. As an illustrative example, the Zic2 protein is selected. Similar to the previous domains, its models learned are depicted in Fig. A4 of the supplementary material. We can observe that cytosines and guanines are highly enriched in the matrix models as well as the scatter plots between nucleotide fractions and model fitnesses ( $p=0.00035$ and $p=0.0072$, respectively). It is consistent with the previous studies that cytosines and guanines are the core binding nucleotides required for zinc finger DNA binding [43]. Nonetheless, we would like to note that zinc finger domains usually bind to DNA sequences in multiple units. Such biological context information cannot be captured by PBM technology.

\section{REAL WORLD APPLICATIONS}

\section{A. PBM Rotation Testing}

We usually have two PBM array datasets for each protein of interest for control. To test the accuracy of the models learned using kmerGA, we can apply each model to its corresponding replicate alternatively; for instance, a model learned on array \#1 can be applied to rank the probes on array \#2 and vice versa. Especially, we are interested in the abilities of the models learned using kmerGA to predict positive probes among all the available probes in an array dataset. Thus we have adopted the traditional measure to define positive probes on each dataset [16]. Mathematically, we define a positive probe to be the probe $y$ whose normalized signal intensity $m_{y}>m i+4 \sigma$, where $m i$ and $\sigma$ are the median and the median absolute deviation (MAD) of all the probe normalized intensities in the same dataset divided by 0.6745 (the MAD of the unit normal distribution), respectively. Following that definition, we have tested the models learned using kmerGA on the previous benchmark PBM datasets. We applied the models to perform the binary classification tasks on the corresponding datasets. In particular, we use a sliding window (with the same length as the learned model of the protein of interest) to scan each sequence and adopt the maximal score as the score of each sequence. Mathematically, given a DNA sequence $D=d_{1} d_{2} d_{3} \ldots d_{T}$ and the corresponding model learned $M$, we compute its predicted score $B_{M}(D)$ as

$$
\begin{aligned}
B_{M}(D) & =\max _{p} S_{M}\left(d_{p} d_{p+1} d_{p+2} \cdots d_{p+L-1}\right) \\
\forall p & \in\{1,2, \ldots, T-L+1\}
\end{aligned}
$$

where $S_{M}\left(d_{p} d_{p+1} d_{p+2} \cdots d_{p+L-1}\right)$ is the function $S$ described in the background section.

The area under curve (AUC) of receiver operating characteristics (ROCs) curve is adopted as the performance metric to estimate the models' accuracies which are depicted in Fig. 7.

From the results, it can be observed that the models learned using kmerGA usually show good performance in predicting positive probes on another array dataset. Comparing to DE and CGA, most of the AUC values achieved by kmerGA are above 0.5 which is the baseline performance value, demonstrating the utility of the models learned using kmerGA. It also implies PBM array data consistency.

\section{B. ChIP-Seq Peak Sequence Predictions}

To demonstrate the utility of the models we have learned using kmerGA further, we have followed the past literature to apply the models to predict ChIP-Seq peak sequences among a set of genomic sequences [44]. In particular, we have checked which models we have learned from the mouse PBM datasets are found in the ENCODE database (K562 cell line), resulting in the following proteins of interests, namely Egr1, Mafk, and Max. The Egrl protein is called early growth response protein 1, a zinc finger protein encoded by Egr1 gene in mammalian genomes [45]. It is a nuclear protein for regulating cell differentiation and mitogenesis, which is also suggested to be involved in different cancers [46]. In contrast, the Mafk protein is a relatively unknown basic leucine zipper TF responsible for developmentally regulated expression of the globin genes [47]. The third one is the Max protein which is a member of the 
bHLH leucine zipper family of TFs. It can interact with several other proteins including the oncoprotein, Myc [48].

Following the convention in [44], we have obtained the ChIP-Seq peak sequences from their BED files in the ENCODE database. After that, we randomly sample equal amounts of sequences with the same lengths as the peak sequences such that a ChIP-Seq peak sequence dataset is obtained for each protein of interest. In each dataset, half of the sequences are the peak sequences from the ENCODE database while the other half are the background sequences randomly sampled.

After the ChIP-Seq peak sequence datasets are obtained, similar to the previous section, we applied the models learned using kmerGA to perform the binary classification tasks on the ChIP-Seq peak sequence datasets using $B_{M}(D)$. Having scanned the datasets, we checked our predictions with the actual peak labels. The results are depicted as ROC curves in Fig. 8. It can be observed that the models learned using kmerGA are found beneficial for the proteins of interests in predicting peak sequences (above baseline). Nonetheless, kmerGA performs differently on different datasets; for instance, as expected, the models learned on the Zbtb3 PBM dataset are just marginally better than the baseline. It can actually be foreseen because the in vitro PBM technology limits itself to $k$-mer enumeration which is not suitable for the zinc finger family, including the Zbtb3 protein, since zinc finger proteins usually bind to DNA in tandem in vivo.

\section{CONCLUSION}

In recent years, high-throughput biotechnologies enable the massive generation of real life data. In particular, PBM allows us to measure the binding specificities of DNA-binding proteins comprehensively. To navigate and extract informative binding site information from PBM data, we have proposed and described evolutionary computation methods to be applied to evolve and learn binding site models which can accurately reflect the ranking of top binding sites (i.e., top aligned $k$-mers). We have shown that the evolutionary computation methods have better performance than the interior point method, one of the best numerical optimization methods nowadays. In addition, the huge population of the models learned along with evolutionary computation methods can enable better understandings on DNA-binding mechanism.

As an illustrative example, we have proposed and described a novel method, namely kmerGA. It assumes and exploits PBM data properties to achieve competitive results on more than 200 datasets. The robustness of kmerGA has also been demonstrated using convergence analysis and parameter analysis. In particular, the accumulated population of the models learned using kmerGA after multiple runs enable case studies to be carried out to reveal novel insights into how different proteins bind to DNA sequences. Two real world applications have been proposed and described to demonstrate the utility of the models learned by kmerGA. The first one is to test the PBM data consistencies using the models learned, while the second one is to apply the models to predict ChIP-Seq peak sequences from other sequences.
Although the above, we would like to note that our performance results should not be over-interpreted to other motif discovery studies since the current problem formulation is different from the past literature reviewed in Section I. The potential drawback is that it relies on a sliding window to segment DNA probe sequences into individual $k$-mers, which may lose the sequence context information. We expect such an limitation will be alleviated when a future improved PBM technology can generate binding affinities for longer probes (i.e., higher $k$ value). Similar to other evolutionary algorithms, the proposed method (kmerGA) is stochastic in nature. Therefore, we suggest multiple runs of kmerGA to avoid any suboptimal convergence.

In the future, predicting the measured binding affinities will be an interesting direction, should the PBM technology be improved to be more noise-free and platform-independent than the current form.

\section{ACKNOWLEDGMENT}

The authors would like to thank Morris Laboratory and Bulyk Laboratory for making their PBM data publicly available and T.-M. Chan for his help in literature review. They would also like to thank the anonymous reviewers for their time.

\section{REFERENCES}

[1] K. C. Wong, Y. Li, C. Peng, A. M. Moses, and Z. Zhang, "Computational learning on specificity-determining residue-nucleotide interactions," Nucleic Acids Res., vol. 43, no. 21, pp. 10180-10189, Dec. 2015.

[2] K. C. Wong, Y. Li, C. Peng, and Z. Zhang, "SignalSpider: Probabilistic pattern discovery on multiple normalized ChIP-Seq signal profiles," Bioinformatics, vol. 31, no. 1, pp. 17-24, Jan. 2015.

[3] K. C. Wong, Y. Li, and C. Peng, "Identification of coupling DNA motif pairs on long-range chromatin interactions in human K562 cells," Bioinformatics, pp. 1-4, Sep. 2015. [Online]. Available: http://bioinformatics.oxfordjournals.org/content/early/2015/ 10/15/bioinformatics.btv555

[4] J. D. Hughes, P. W. Estep, S. Tavazoie, and G. M. Church, "Computational identification of cis-regulatory elements associated with groups of functionally related genes in Saccharomyces cerevisiae," J. Mol. Biol., vol. 296, no. 5, pp. 1205-1214, Mar. 2000.

[5] T. L. Bailey and C. Elkan, "The value of prior knowledge in discovering motifs with MEME," in Proc. Int. Conf. Intell. Syst. Mol. Biol., vol. 3. Cambridge, U.K., 1995, pp. 21-29.

[6] D. Che, Y. Song, and K. Rasheed, "MDGA: Motif discovery using a genetic algorithm," in Proc. 7th Annu. Conf. Genet. Evol. Comput., Washington, DC, USA, 2005, pp. 447-452.

[7] M. Kaya, "MOGAMOD: Multi-objective genetic algorithm for motif discovery," Expert Syst. Appl., vol. 36, no. 2, pp. 1039-1047, 2009.

[8] L. Li, "GADEM: A genetic algorithm guided formation of spaced dyads coupled with an EM algorithm for motif discovery," J. Comput. Biol., vol. 16, no. 2, pp. 317-329, 2009.

[9] F. F. M. Liu, J. J. P. Tsai, R.-M. Chen, S. N. Chen, and S. H. Shih, "FMGA: Finding motifs by genetic algorithm," in Proc. 4th IEEE Symp. Bioinform. Bioeng., Taichung, Taiwan, 2004, pp. 459-466.

[10] Z. Wei and S. T. Jensen, "GAME: Detecting cis-regulatory elements using a genetic algorithm," Bioinformatics, vol. 22, no. 13, pp. $1577-1584,2006$.

[11] M. A. Lones and A. M. Tyrrell, "Regulatory motif discovery using a population clustering evolutionary algorithm," IEEE/ACM Trans. Comput. Biol. Bioinform., vol. 4, no. 3, pp. 403-414, Jul./Sep. 2007.

[12] T.-M. Chan, K.-S. Leung, and K.-H. Lee, "TFBS identification based on genetic algorithm with combined representations and adaptive postprocessing," Bioinformatics, vol. 24, no. 3, pp. 341-349, Feb. 2008.

[13] M. Tompa et al., "Assessing computational tools for the discovery of transcription factor binding sites," Nat. Biotechnol., vol. 23, no. 1, pp. 137-144, 2005. 
[14] M. F. Berger et al., "Compact, universal DNA microarrays to comprehensively determine transcription-factor binding site specificities," Nat. Biotechnol., vol. 24, no. 11, pp. 1429-1435, Nov. 2006.

[15] K. Robasky and M. L. Bulyk, "UniPROBE, update 2011: Expanded content and search tools in the online database of protein-binding microarray data on protein-DNA interactions," Nucleic Acids Res., vol. 39, pp. D124-D128, Jan. 2011.

[16] X. Chen, T. R. Hughes, and Q. Morris, "RankMotif++: A motifsearch algorithm that accounts for relative ranks of K-mers in binding transcription factors," Bioinformatics, vol. 23, no. 13, pp. i72-i79, Jul. 2007.

[17] B. C. Foat, S. S. Houshmandi, W. M. Olivas, and H. J. Bussemaker, "Profiling condition-specific, genome-wide regulation of mRNA stability in yeast," in Proc. Natl. Acad. Sci. USA, vol. 102, no. 49, pp. 17675-17680, Dec. 2005.

[18] X. S. Liu, D. L. Brutlag, and J. S. Liu, "An algorithm for finding proteinDNA binding sites with applications to chromatin-immunoprecipitation microarray experiments," Nat. Biotechnol., vol. 20, no. 8, pp. 835-839, Aug. 2002.

[19] A. Tanay, "Extensive low-affinity transcriptional interactions in the yeast genome," Genome Res., vol. 16, no. 8, pp. 962-972, Aug. 2006.

[20] K.-C. Wong, C. Peng, Y. Li, and T.-M. Chan, "Herd clustering: A synergistic data clustering approach using collective intelligence," Appl. Soft Comput., vol. 23, pp. 61-75, Dec. 2014.

[21] K. C. Wong, T. M. Chan, C. Peng, Y. Li, and Z. Zhang, "DNA motif elucidation using belief propagation," Nucleic Acids Res., vol. 41, no. 16, p. e153, Sep. 2013.

[22] C. Zhang and A. K. C. Wong, "Toward efficient multiple molecular sequence alignment: A system of genetic algorithm and dynamic programming," IEEE Trans. Syst., Man, Cybern. B, Cybern., vol. 27, no. 6, pp. 918-932, Dec. 1997.

[23] A. M. Moses and S. Sinha, "Regulatory motif analysis," in Bioinformatics: Tools and Applications, D. Edwards, J. Stajich, and D. Hansen, Eds. New York, NY, USA: Springer, 2009, pp. 137-163.

[24] M. Wright, "The interior-point revolution in optimization: History, recent developments, and lasting consequences," Bull. Amer. Math. Soc., vol. 42, no. 1, pp. 39-56, 2005.

[25] D. E. Goldberg, Genetic Algorithms in Search, Optimization and Machine Learning. Reading, MA, USA: Addison-Wesley, 1989.

[26] K. V. Price, "Differential evolution vs. the functions of the 2nd ICEO," in Proc. IEEE Int. Conf. Evol. Comput., Indianapolis, IN, USA, Apr. 1997, pp. 153-157.

[27] R. Thomsen, "Multimodal optimization using crowding-based differential evolution," in Proc. Evol. Comput. Congr. (CEC), vol. 2. Portland, OR, USA, Jun. 2004, pp. 1382-1389.

[28] S. Boyd and L. Vandenberghe, Convex Optimization. Cambridge, U.K.: Cambridge Univ. Press, 2004. [Online]. Available: http://www.amazon.com/Convex-Optimization-Stephen-Boyd/dp/ 0521833787

[29] H. Bersini, M. Dorigo, S. Langerman, G. Seront, and L. Gambardella, "Results of the first international contest on evolutionary optimisation (1st ICEO)," in Proc. IEEE Int. Conf. Evol. Comput., Nagoya, Japan, May 1996, pp. 611-615.

[30] J. Tvrdík, "Adaptation in differential evolution: A numerical comparison," Appl. Soft Comput., vol. 9, no. 3, pp. 1149-1155, Jun. 2009. [Online]. Available: http://dx.doi.org/10.1016/j.asoc.2009.02.010

[31] S.-M. Tse, Y. Liang, K.-S. Leung, K.-H. Lee, and T.-S. Mok, "A memetic algorithm for multiple-drug cancer chemotherapy schedule optimization," IEEE Trans. Syst., Man, Cybern. B, Cybern., vol. 37, no. 1, pp. 84-91, Feb. 2007.

[32] K. Deb and D. E. Goldberg, "An investigation of niche and species formation in genetic function optimization," in Proc. 3rd Int. Conf. Genet. Algorithms, 1989, pp. 42-50.

[33] K.-C. Wong, C. Peng, M.-H. Wong, and K.-S. Leung, "Generalizing and learning protein-DNA binding sequence representations by an evolutionary algorithm," Soft Comput., vol. 15, no. 8, pp. 1631-1642, Aug. 2011. [Online]. Available: http://dx.doi.org/10.1007/s00500-011-0692-5

[34] K. C. Wong and Z. Zhang, "SNPdryad: Predicting deleterious nonsynonymous human SNPS using only orthologous protein sequences," Bioinformatics, vol. 30, no. 8, pp. 1112-1119, 2014.

[35] K. A. De Jong, Evolutionary Computation: A Unified Approach. Cambridge, MA, USA: MIT Press, 2006.

[36] H. G. Cobb, "An investigation into the use of hypermutation as an adaptive operator in genetic algorithms having continuous, time-dependent nonstationary environments," Naval Res. Lab., Washington, DC, USA, Tech. Rep. 6760, 1990
[37] R. Durbin, S. R. Eddy, A. Krogh, and G. Mitchison, Biological Sequence Analysis: Probabilistic Models of Proteins and Nucleic Acids. Cambridge, U.K.: Cambridge Univ. Press, Jul. 1998. [Online]. Available: http://www.amazon.ca/exec/obidos/redirect?tag=citeulike09-20\&amp; path=ASIN/0521629713

[38] K.-C. Wong, C.-H. Wu, R. K. P. Mok, C. Peng, and Z. Zhang, "Evolutionary multimodal optimization using the principle of locality," Inf. Sci., vol. 194, pp. 138-170, Jul. 2012.

[39] Y. S. Ong, P. B. Nair, and A. J. Keane, "Evolutionary optimization of computationally expensive problems via surrogate modeling," AIAA J., vol. 41, no. 4, pp. 687-696, 2003.

[40] Y. Jin, "A comprehensive survey of fitness approximation in evolutionary computation," Soft Comput., vol. 9, no. 1, pp. 3-12, 2005.

[41] G. Badis et al., "Diversity and complexity in DNA recognition by transcription factors," Science, vol. 324, no. 5935, pp. 1720-1723, Jun. 2009.

[42] B. Alberts et al., "Molecular biology of the cell," Ann. Botany, vol. 91, no. 3, pp. 401-410, 2003 .

[43] A. Klug, "The discovery of zinc fingers and their applications in gene regulation and genome manipulation," Аппи. Rev. Biochem., vol. 79, no. 1, pp. 213-231, 2010

[44] J. O. Yanez-Cuna et al., "Dissection of thousands of cell type-specific enhancers identifies dinucleotide repeat motifs as general enhancer features," Genome Res., vol. 24, no. 7, pp. 1147-1156, Jul. 2014.

[45] G. Thiel and G. Cibelli, "Regulation of life and death by the zinc finger transcription factor Egr-1,' J. Cell. Physiol., vol. 193, no. 3, pp. 287-292, 2002.

[46] V. Baron, E. D. Adamson, A. Calogero, G. Ragona, and D. Mercola, "The transcription factor Egr1 is a direct regulator of multiple tumor suppressors including TGF $\beta 1$, PTEN, p53, and fibronectin," Cancer Gene Ther, vol. 13, no. 2, pp. 115-124, 2005.

[47] T. Oyake et al., "Bach proteins belong to a novel family of BTB-basic leucine zipper transcription factors that interact with MafK and regulate transcription through the NF-E2 site," Mol. Cell. Biol., vol. 16, no. 11, pp. 6083-6095, 1996.

[48] D. E. Ayer, L. Kretzner, and R. N. Eisenman, "MAD: A heterodimeric partner for MAX that antagonizes MYC transcriptional activity," Cell, vol. 72, no. 2, pp. 211-222, 1993.

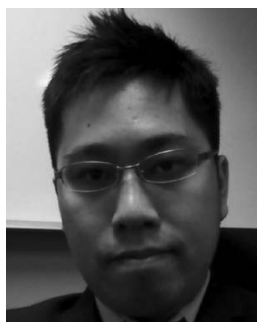

Ka-Chun Wong received the B.Eng. degree in computer engineering from the United College, Chinese University of Hong Kong, Hong Kong, in 2008, the M.Phil. degree from the Department of Computer Science and Engineering, Chinese University of Hong Kong, in 2010, and the Ph.D. degree from the Department of Computer Science, University of Toronto, Toronto, ON, Canada, in 2014.

He has been an Assistant Professor with the City University of Hong Kong, since 2015. His current research interests include bioinformatics, computational biology, evolutionary computation, data mining, machine learning, and interdisciplinary research.

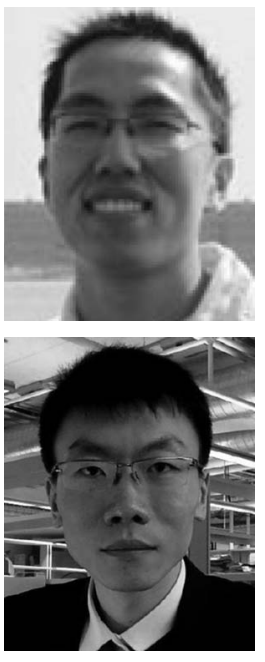

Chengbin Peng received the bachelor's and master's degrees in computer science from Zhejiang University, Hangzhou, China, in 2007 and 2010, respectively, and the Ph.D. degree from the King Abdullah University of Science and Technology, Thuwal, Saudi Arabia, in 2015, with the support from KAUST Ph.D. fellowship, provost's award, and teaching assistantships.

His current research interests include data mining algorithms and complex networks.

Yue Li received the B.Sc. degree (Hons.) in bioinformatics from the University of Saskatchewan, Saskatoon, SK, Canada, in 2010, with minor in statistics, and the M.Sc. degree and the Ph.D. degree in computational biology from the University of Toronto, Toronto, ON, Canada, in 2012 and 2014 respectively.

$\mathrm{He}$ is currently a Post-Doctoral Associate at Computer Science and Artificial Intelligence Laboratory, Massachusetts Institute of Technology, Boston, MA, USA. His current research interests include developing machine-learning methods and bioinformatics tools to reveal meaningful patterns involving genetics, epigenetics, and expression dynamics that are associated with complex human diseases. 